\title{
Combined ketogenic diet and walking exercise interventions in community older groups
}

\author{
Jia-Ping $\mathbf{W} \mathbf{u}^{1,2}$ \\ ${ }^{1}$ Research Center for Healthcare Industry Innovation, National Taipei University \\ of Nursing and Health Sciences, Taipei City, R.O.C. \\ ${ }^{2}$ Medcom Biotech co., Ltd., Taipei City, R.O.C.

\section{Correspond author: Jia-Ping $\mathbf{W u}^{1,2}$} \\ ${ }^{1}$ Research Center for Healthcare Industry Innovation, National Taipei University \\ of Nursing and Health Sciences. No. 365, Mingde Rd., Beitou Dist., Taipei City \\ 11219, Taiwan, R.O.C. \\ ${ }^{2}$ Medcom Biotech co., Ltd. Rm. 3, 18 F., No. 237-1, Minquan W. Rd., Datong \\ Dist., Taipei City 10357, Taiwan, R.O.C. \\ EMAIL: affymax0823@yahoo.com.tw
}

\begin{abstract}
The ketogenic diet and walking exercise training activity are two key public health lifestyle factors. The potential of combined lifestyle factors interventions focused on getting to compliance in forced exercise. A balanced ketogenic diet and regular exercise activity is a key modifiable factor to the prevention and management of chronic diseases. Influence health across the lifespan and reduction of the risk of premature death through several biological mechanisms. Community older group's lifestyle factors interventions contribute identity in their natural living environment. While the older health benefits of walking exercise training strategies are commonly to study, combined ketogenic diet and walking exercise interventions have induced greater benefits in community older groups.
\end{abstract}

Keywords: ketogenic diet, exercise therapy, community health planning, natural, exercise intervention.

\section{Introduction}

The ketogenic diet is a $60 \%$ high-fat, $30 \%$ adequate-protein, and $10 \%$ lowcarbohydrate diet used to treat aging-related diseases in the community older groups. The ketogenic diet interventions are a specialized diet that involves a highly restricted intake of carbohydrates and proteins and a high proportion of 
fat consumption in community older groups [1]. It has proven to be used in the treatment of older men-related diseases in community groups because the mechanism of action of the ketogenic diet interventions causes changes in the levels of ketone bodies with exercise training interventions in the body, reducing the aging-related diseases [2,3]. The purpose of this review was to systematically review the systemic effects of ketogenic diet restriction when combined with walking exercise intervention in community older groups. Thus, in this review, we want to discuss the combined ketogenic diet and walking exercise interventions in community older groups. The ketogenic diets very high in fat can promote ketogenesis differently depending on other different macronutrient ratios [4]. The ketogenic diets for weight loss in older humans may be counterproductive to obesity, which is not typically associated with NAFLD/NASH [5,6]. Acetoacetate, acetone, and $\beta$-hydroxybutyrate are the three ketone bodies produced in community older groups. It's also important to eat a healthy ketogenic diet and exercise interventions regularly as well as check in with your healthcare provider [7]. After sed short-time walking exercise, make appropriate adjustments based on your own feelings such as tiredness and sleepiness [8]. However, the benefits of Walking exercise regimens improve the immune system, helps digestion, promote the release of muscle hormones, and when they enter the body to eliminate inflammation, reduce visceral fat, reduce inflammation, helps improve brain-derived neurotrophic factor substances, mitochondrial cells work normally, and help longevity $[9,10-$ 12]. The precise regimen of action of the combined ketogenic diet and walking exercise interventions in community older groups is not known, although many possible explanations have been proposed. There are many changes that occur in the body as a result of the ketogenic diet, but it is unclear which of these alterations is responsible for the walking exercise training interventions effects. This is expected, however, as the mechanism of action of the combined ketogenic diet and walking exercise interventions in community older groups is similarly a mystery.

\section{The ketogenic diet in the community older groups.}

The key aspect of ketogenic diet is a high proportion of fats, adequate levels of protein, a low proportion of carbohydrates primarily used to treatment difficultto-control aging chronic diseases [13]. The ketogenic diet is now used to treat in the community older groups for rapidly burn more fat when there is a low carbohydrate intake [14]. The ketogenic diet, low carbohydrate intake can lead 
to elevated blood ketone bodies. Measured blood ketones levels can allow for adjustment of the ketogenic diet to meet the user's needs [15]. But now new technologies are being researched in the breath acetone sensors are becoming more popular due to less invasiveness and convenience [16,17-19]. Future technologies are very promising but are still in the early development stages. The ketogenic diet became popular as a therapy for epilepsy in the 1920s and 30s. Recently, it was developed to provide an alternative to anti-aging, which had demonstrated success as an aging therapy [20]. However, the ketogenic diet interventions are eventually largely abandoned due to the mitochondrial dysfunction and excessive inflammatory responses to induce pathology in agerelated diseases in the community older groups. There are several theories about the mechanism of action of the ketogenic diet intervention, including increased acidity in the blood.

\section{Ketogenic diet is converted to ketone bodies.}

The ketogenic diets forces to burn off of fats rather than carbohydrates [21]. A ketogenic diet, a high fat, in food is converted triglyceride (TG). The liver converts triacylglycerol (TAG) into fatty acid and ketone bodies. An elevated ketone bodies in the blood eventually lowers the aging-related diseases [22]. We hoped that ketogenic diet therapy could be maintained ketone bodies by the liver in the community older groups. Blood ketone bodies were produced $\beta$ hydroxybutyrate, acetoacetate, and acetone. They consumed a very lowcarbohydrate, and excess high-fat diet [23]. Ketone bodies (KBs) are considered as an alternative source of energy supply [24]. When a person eats a regular ketogenic diet, food is converted into glucose, which is transported around the body and used by various cells as an energy source [25], but when too little carbohydrates are available, the liver processes fats to provide the brain with energy in the form of fatty acids and ketone bodies. An increased blood level of ketone bodies is referred to as ketosis. These ketone bodies are thought to possess anti-aging properties in the community older groups, as $\beta$ hydroxybutyrate supplementary has been shown to protect old human health [26]. In 1921, endocrinologists demonstrated that ketone bodies were produced by the liver including three water-soluble compounds, acetone, $\beta$ hydroxybutyrate, and acetoacetate, as they eat a diet rich in fat and low in carbohydrates. 


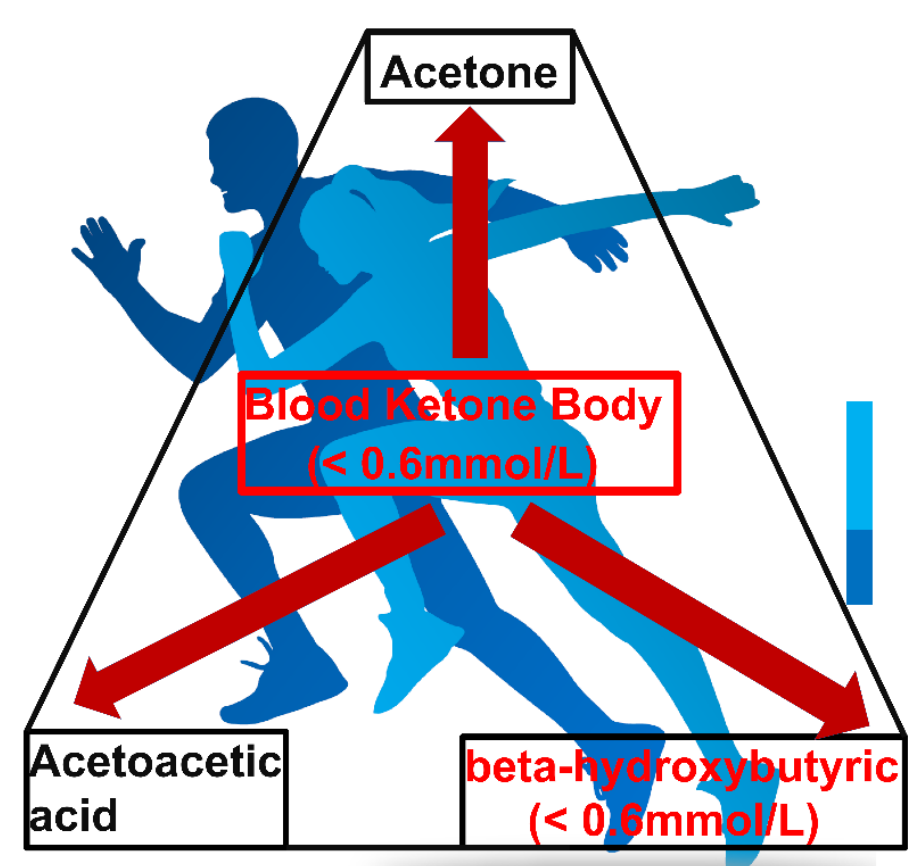

Figure 1. Blood ketone body converses. Ketogenic diet raised ketone body levels. Blood ketone bodies ( $<0.6 \mathrm{mmole} / \mathrm{L}$ ) are markers specifically $\beta$ hydroxybutyrate $(\mathrm{BHB})$, acetoacetate $(\mathrm{AcAc})$, and acetone. The breath acetone level is lower compared to blood BHB. Direct measurement of beta hydroxybutyrate circumvents this problem. Therefore, the $\beta$-hydroxybutyrate $(\mathrm{BHB})$ blood test may underestimate the true circulating ketone bodies.

The key aspect of the ketogenic diet involves the restriction of carbohydrates, which are no longer able to be converted to glucose and provide for the body's metabolic and energy needs [27]. To compensate for this, fatty acids are converted into fuel sources through a process of oxidation in the mitochondria. To detect acetoacetate in blood, but does not react with $\beta$-hydroxybutyrate which is the predominant circulating ketone body. In the community older groups' bodies can become more strongly positive as the metabolic derangements improve $\beta$-hydroxybutyrate is converted to acetoacetate [28]. The ketogenic diet mimics aspects of starvation, the body is forcing to burn fats rather than carbohydrates, when this is combined with a low intake of carbohydrates which causes the body to produce ketones [29]. The stabilization of the ketogenic diet may occur as a result of the efficiency of the ketone bodies as a fuel source. The ketogenic diet is converted fatty acids to ketone bodies for energy to increase the number of mitochondria as the body adapts [30]. However, this is of no consequence provided the ketogenic diet converted 
ketone bodies ( $\beta$-hydroxybutyrate and acetoacetate) are closing in community older groups and the patient is continuing to improve clinically.

\section{The $\beta$-hydroxybutyrate (BHB) ketone supplements interventions in the community older groups.}

Furthermore, we discuss the role of $\beta$-hydroxybutyrate (BHB) supplementary interventions exercise factors released by the liver [31]. Walking exercise training may be able to increase their blood $\beta$-hydroxybutyrate (BHB) concentrations in the community older groups and be increased in ketosis. Endogenous production of high levels of the ketone body $\beta$-hydroxybutyrate $(\mathrm{BHB})$ is regarded as $5 \mathrm{mM}$ blood $\mathrm{BHB}$ for $120 \mathrm{~min}$ after walking exercise in the older men [32]. This ketogenic diet has long been used as a treatment in the community of older men focused on the therapeutic effects of the ketone body $\beta$-hydroxybutyrate (BHB). Recent reports demonstrate that developed ketone can help significantly increase the blood circulating $\beta$-hydroxybutyrate in the community older humans [33]. Ketone supplements can efficiently attenuate age-related diseases in older humans. We argue this inflection point affects older human health. Some reports indicated that one of the ketone bodies, $\beta$ hydroxybutyrate $(\mathrm{BHB})$, in the community older humans can inhibit agingrelated diseases such as sarcopenia or Alzheimer disease (AD) [34]. The favorable aspect of ketosis in both ketogenic diet and ketogenic supplements in aging-related diseases has been discussed. We summarize and suggest that aging research is entering a new milestone that has unique medical, commercial, and societal implications.

\section{The different types of ketogenic diet intervention regimens in the community older groups.}

The ketogenic diet intervention regimens are a special diet designed to help the community older groups that fail to respond adequately to aging-related diseases [35]. In the absence of glucose due to lack of carbohydrates in the ketogenic diet interventions, the community older groups are no longer able to be converted to glucose and provide the body's metabolic and energy needs, fatty acids are the majored converted into the fuel sources through synthesized the ketone bodies $\beta$-hydroxybutyrate, acetoacetate and acetone [36,37]. The ketogenic diet is a mixed diet containing low carbohydrates, consisting primarily of proteins and fat. Some healthy foods are eaten on a ketogenic diet, for 
example, seafood, low-carb vegetables, cheese, eggs, meat, poultry, coffee, and tea [38]. The importance of high fat in aging-related diseases reducing regimens on different walking exercise training models is shown by comparing the effects of four different types of ketogenic dietary regimens. A typical ketogenic diet interventions regimens are made up of the following: ( I ). A standard keto diet (SKD): Typically contains a very low, only $5 \%$ carbohydrate, $15 \%$ moderate proteins, $80 \%$ high fat diet. This classic SKD contains a 3:1 ratio to combined protein and carbohydrate. (II ). The high protein keto diet (HPKD): This contains $5 \%$ carbohydrates, $35 \%$ protein, and $60 \%$ fat. HPKD is about the same as the standard keto diet but includes more protein. (III). The cyclical keto diet (CKD): This ketogenic diet feeds like 5 ketogenic days of periods of highercarbs feeds, and then 2 high carbohydrate days. (IV). The targeted keto diet (TKD): This type of ketogenic diet allows you to add more around carbohydrates workouts. Although this keto diet is usually safe for diabetes, epilepsy, and aging-related diseases, they may be had some initial body adaptation. Be sure to consume a balanced optimized ketogenic diet to support your fitness program. All food groups are necessary to sustain healthy energy levels and get the most out of your workout [39]. A ketogenic diet contains 5\% carbohydrates, carbohydrates are vital, as they can fuel your muscles before exercise [40]. Carbohydrates are also important after walking exercise training to replenish glycogen stores and assist with the absorption of amino acids into your muscles during recovery [41]. 35\% protein helps improve muscle recovery after walking exercise training, repairs tissue damage, and builds muscle mass [42]. $60 \%$ consuming healthy fats has been shown to help burn body fat and preserve muscle fuel during workouts, making your energy last longer [43]. The ketogenic diet interventions contain adequate amounts of protein for body growth. The total protein in the ketogenic diet is also sufficient to maintain health for a given older age. In the classic ketogenic diet, the ratio of fats to carbohydrates and proteins combined is $4: 1$ [44]. Although it emerged in the community older groups of aging-related diseases could be effectively controlled using these interventions. They may still fail to achieve aging control in the community older groups [45]. For these intervention individuals, the ketogenic diet interventions were re-introduced as a technique for managing the condition. However, the ketogenic diet has been shown in a study of rats to have anti-aging properties and inhibit the development of aging-related diseases in the community older groups.

\section{Walking exercise training in the older community groups.}


Exercise training can help lower the risk of aging-related diseases in the older community groups, for example, decreases blood pressure, lower LDL cholesterol levels, developing type 2 diabetes, increase your heart's size and strength, and improve cardiorespiratory fitness [46]. Walking exercise training is a low-intensity aerobic activity that reduces the risk of the older community groups' diseases [47]. If you have another aging-related chronic disease, you should speak with your healthcare professional before starting a new exercise program. The difference of intensity of walking exercise performs change arterial system during the exercise stimulus [48]. Moderate walking exercise training models can improve arterial endothelial function in the community group of an older healthy man. General recommendations to promote good overall health, aim to get at least 150 minutes of moderate-intensity exercise, or 75 minutes of high-intensity exercise training, or a combination of the two each week for optimal young adult health [49]. However, low-intensity walking exercise training for 15 minutes at least 3 times per week and spend 10 minutes of your lunch break walking exercise. Chronic exercise training that can mimic the effects of exercise is associated with lower blood pressure response in older men [50]. Starting a new walking exercise routine can be challenging in the community older groups. However, having real objectives can help you maintain a fitness program in the long term [51]. Simply it is important to warm up before you start your walking exercise like arm swings, leg kicks, and walking lunges doing so can help prevent injuries and improve your flexibility and reduce soreness [52]. Alternatively, walking exercise training in the older community groups warm up by doing easy movements of the walking exercise training you're planning to do. For example, warm-up before you walking exercise.

\section{The walking exercise intervention in the community older groups.}

Walking exercise training interventional improvements oxygen consumption between 15 and 29\% in older adults lasting between 6 and 12 months [53]. A significant improvement in aerobic capacity was also shown following exercise training of shorter duration almost 9 12 weeks in older people (Figure 2). A time course, intensity, and adaptation in maximal aerobic capacity with walking exercise training are different in older compared with younger people and suggest improvements in both cardiac function and peripheral muscles oxygen extraction [54]. During exercise training, oxygen consumption in older people is higher than in people. The successful elderly Walking exercise interventions 
regimens. The successful elderly walking exercise regimens are a limited effect on arterial structural remodeling $[55,56]$. Walking exercise has major implications on endothelial function and endothelium dilation [57]. Therefore, walking exercise significantly improves endothelial flow-mediated dilation function. Other reports demonstrated that endothelium dilation is greater in older man. One hundred days of walking exercise intervention improves endothelium dilation in the older healthy men [58]. The greater endothelium dilation in older men who regularly perform aerobic exercise is mediated nitric oxide. The intensity of exercise performed and duration of the exercise stimulus may be changed the arterial system [59]. However, no change in endothelial function is observed for mild or high-intensity exercise training for 12 weeks in a group of young healthy men. In a healthy older population a simple walking exercise did not improve endothelial function. Walking exercise interventions of a shorter duration do not alter the endothelial function or arterial stiffness in the older population, for example, 10 days [60]. It is possible that high exercise intensity could diminish oxidative stress. Based on this study regimen it is reasonable to suggest that at least 90 days of exercise training is necessary to stimulate improvements in the elderly endothelial function [61]. A daily brisk walking exercise intervention for 120 days was associated with significantly improved arterial compliance in the older community groups [62]. Regular exercise intervention training is independent of baseline compliance body composition and oxygen capacity [63]. There are many different types of walking exercise training to choose from interventions. Find a new regiment nice for you and be sure to vary them occasionally in the community older groups, for example walking speed over $4 \mathrm{~m}$ walking distance in $\mathrm{m} / \mathrm{s}$. The goal is to start to help prevent injuries slowly to build up your fitness level and let your body rest from time to time [64]. Keeping track of your walking exercise training progress in the community older groups or taking a virtual group class are examples of actionable steps that can help you stay motivated and achieve your goals. From an early treatise collection, authors also describe how an exercising old man was cured of aging-related diseases when he was completed from consuming a ketogenic diet [65]. Neither walking exercise intervention nor the ketogenic diet intervention is able to cure aging but work due to their ability to suppress age-related diseases. This session describes how alterations in the walking exercise intervention and ketogenic diet intervention played a role in anti-aging management. Forced the elderly walking exercise regimen during 120 day's timelines in the community older groups (Figure 2). This timeline details the important events of each phase of the 
elderly walking exercise regimen during each day of the study. The pre-exercise phase during 50 to 60 days. This stage is the pre-acclimation phase involves the older men's experimenter handling and baseline locomotor activity.

Stage 1: The older human experimenter handling, 2 5 min/day, 25 days.

Stage 2: The baseline locomotor activity, $60 \mathrm{~min} /$ day, 35 days.

\section{The elderly Walking exercise regimens}

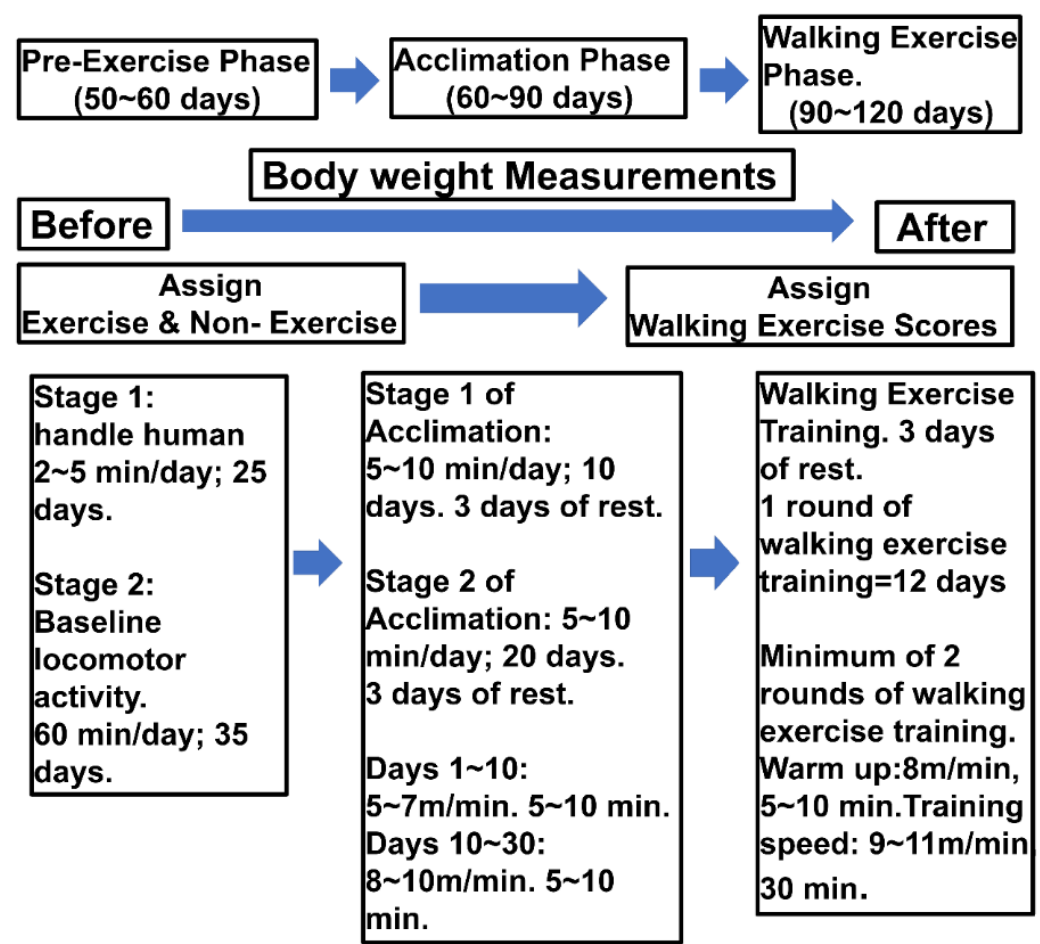

Figure 2. The successful elderly walking exercise regimens in the community older groups. This elderly walking exercise is an easy-to-follow program. This program can be adjusted to your fitness level and made as challenging as you want. One round of walking exercise training will only take you 12 days, and one day will only take you 30 minutes to complete. Doesn't require equipment.

During the acclimation phase (60 90 days) all older humans undergo 10 days of acclimation walking exercise training.

Stage 1 of acclimation phase: 5 10 $\mathrm{min} /$ day, 10 days, $5 \sim 7 \mathrm{~m} / \mathrm{min}, 5 \sim 10 \mathrm{~min}$, by 3 days of rest.

Stage 2 of acclimation phase: 5 10 $\mathrm{min} /$ day, 20 days, $8 \sim 10 \mathrm{~m} / \mathrm{min}, 5 \sim 10 \mathrm{~min}$, by 3 days of rest.

During the walking exercise training phase (90 120 days), one round of walking 
exercise training needs 12 consecutive days. A minimum of 2 rounds of walking exercise training followed by a 6 -days rest period is required during the walking exercise training phase (24 days). Furthermore, this regimen can be modified to include multiple rounds of walking exercise training in this phase. Bodyweight measurements can be made throughout all phases of the study a before and after each phase of this walking exercise training regimen. Assigned nonexercise and walking exercise training sessions scores after all acclimation and walking exercise training phase scores, and range from 1 4, with 4 being the highest possible score. Briefly,

1. Assign a training score of 4 : the older human walking exercise entire walking training session without assistance.

2. Assign a training score of 3 : the older human walking exercise entire walking training requires minimal assistance (less than $25 \%$ ) from the regimen.

3. Assign a training score of 2 : the older human walking exercise require much assistance (greater than $25 \%$ ) from the regimen.

Finally, a training score of 1: the older human walking exercise are noncompliant and fail to complete an exercise session.

\section{Combined ketogenic diet and walking exercise interventions in the older community groups.}

Combined a ketogenic diet with walking exercise interventions is one of the most effective ways to reduce the risk of aging-related diseases in the older community groups [66]. The ketogenic diet and walking exercise are both important for optimal health. Both ketogenic diet and walking exercise interventions in the older community groups can help reduce aging-related heart, brain, vascular, stomach, muscle, lung, liver, kidney, and large intestine injury (Figure 3 ). While old men may be tempted to pick one over the other, a ketogenic diet and walking exercise training work hand in hand, and combining both will optimize health and quality of life [67]. Cardiac physiological functions are associated with walking exercise training intervention. After one year of progressive walking exercise training intervention was confirming physiological cardiac remodeling with walking exercise intervention in the community older people. The influence of walking exercise interventions on aging-related cardiovascular diseases demonstrates in older men than young. In older community groups exhibited myocardial fatty acid metabolism response to beta-adrenergic stimulation after 12 months of walking exercise training [68]. The well-established ketogenic diet promotes the older man's health. The 
ketogenic diet interventions are high in healthy unsaturated fats from undergoing walking exercise interventions in later life. The ketogenic diet and walking exercise intervention change energy metabolism effect on heart, brain, vascular, stomach, muscle, lung, liver, kidney, and large intestine injury $[69,70]$.

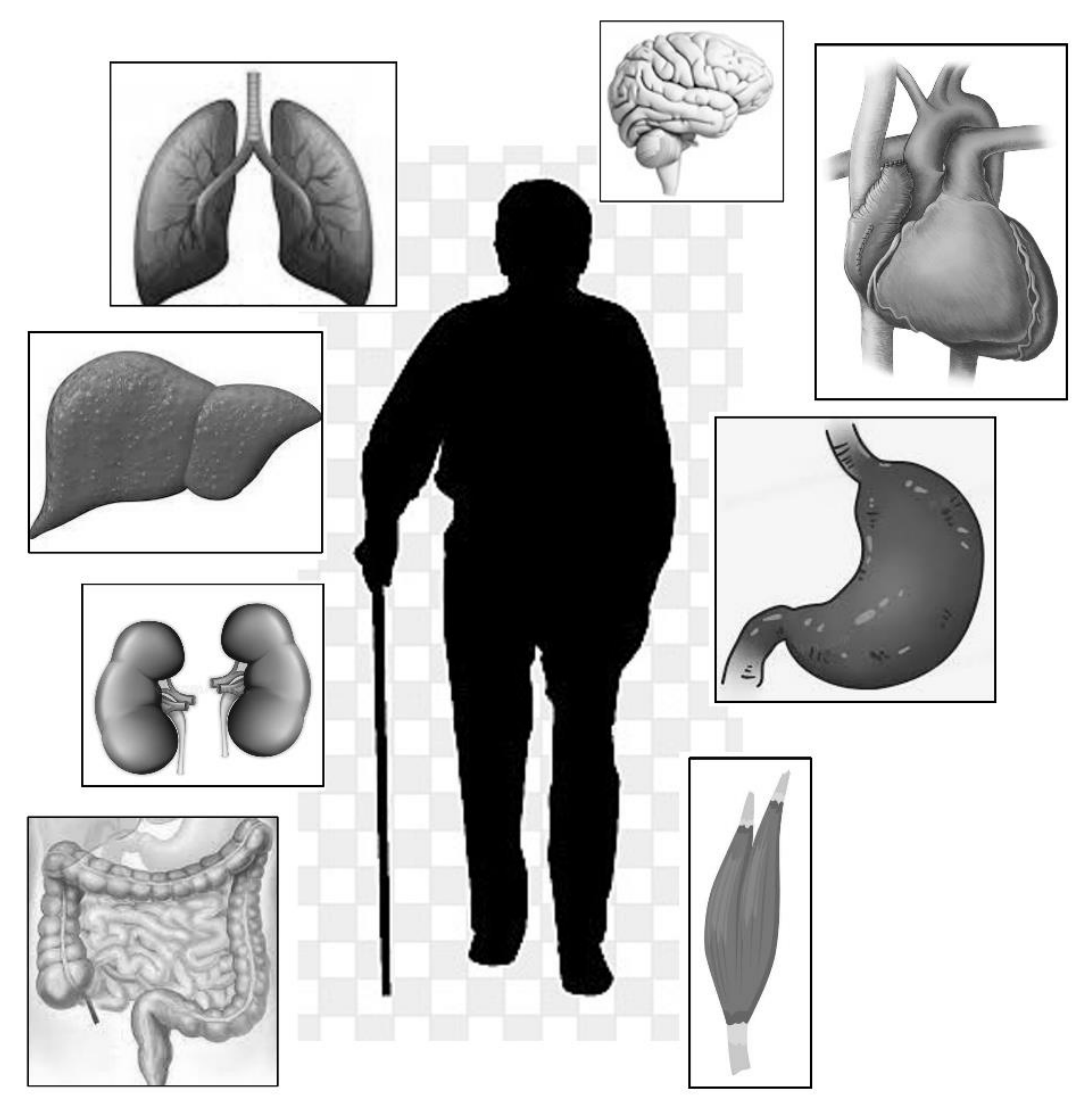

Figure 3. Combined ketogenic diet and exercise interventions in community older groups. The foods of the ketogenic diet we eat can support or hinder older health. The different intensity exercise interventions combined with the ketogenic diet have different effects on the older man's health.

Ketogenic diets among non-pharmacological treatments for those with exercise intolerance are available to the brain, muscle, and heart, where they generate energy for cells in the mitochondria [71]. The major aging-related heart diseases pathophysiological conditions: left ventricular hypertrophy, chronic heart failure, atrial fibrillation, arterial structural remodeling [72]. Pathophysiology is related to multifactorial interventions other than diet or supplementation. In the community older human groups treated with difficultto-control syndromes are those requiring a lot of energy such as heart, brain, and muscle [73]. The brain in a carbohydrate-rich diet usually relies on glucose as the preferred substrate for an energy source. The ketogenic diet is a special 
case of a high-fat diet, about adopting saturated fat in the diet as a cause of heart disease in the community older groups, the long-term ketogenic diet might decrease mitochondrial functions [74]. Glucose is initially the context of a low carbohydrate catabolized in the cytoplasm through the process of glycolysis which produces ATP and NADH [75]. The ketogenic diet reduces hyperglycemia and hyperinsulinemia. Amino acids of threonine, isoleucine, leucine, and lysine were observed for ketogenic amino acids is not true for the heart, conversely, the anoxic heart experiences the greatest [76]. Combined ketogenic diet and exercise interventions in community older groups are high in healthy unsaturated fats from olive oil manipulate nutrient-sensing pathways, particularly heart infarction, diabetes mellitus, and also liver, lung, and kidney disease varieties and antioxidants that help fight harmful molecules free radicals.

\section{Molecular and cellular of the combined ketogenic diet and walking exercise interventions in the community older groups.}

The physiological molecular and cellular mechanisms of the combined ketogenic diet and walking exercise interventions in the older community groups that underlie diminished aging response in older age (Table 1). 120 days of walking exercise training interventions produced a reduction in plasmatic levels of protein carbonylation and lipid peroxidation in older [77]. Lipid peroxidation is one of the most irreversible changes of oxidative protein modifications, observed on an increase in the protein carbonylation and lipid peroxidation in the community older groups [78-80]. However, nonpharmacological strategies such as exercise interventions and ketone body supplements are of significant difference decreased. In the combined ketogenic diet and walking exercise interventions in the older community groups reduced nucleic acid oxidation and lipid peroxidation were observed [80, 81-83]. Ketone body supplementation and walking exercise interventions have been shown to result in a reduction in superoxide dismutase (Mn-SOD) levels [84]. While 120 days of the walking training exercise was seen to be associated with increased SOD activity. The earliest studies showed glutathione reductase [85,86], catalase [87,88], glutamine synthetase [89] that these compounds cause older lifestyle changes like you know, people talk about exercising and walking that 
improve your health for your body, and managing stress, among participants give lifestyle tips on the ketogenic diet and walking exercise training to control their mitochondria keep moving.

\section{Table1: Oxidative disturbances are found in the community older groups.}

Target

Protein carbonylation

Lipid peroxidation

Nucleic acid oxidation

Mn SOD

Glutathione reductase

Catalase

Glutamine synthetase

NO synthase
Modification References

increased

[77-80]

increased

[78-80]

increased

$[80,81-83]$

increased

$[84,85]$

decreased

$[85,86,87]$

decreased

$[87,88]$

decreased

increased
[89]

[90]

After exercise interventions, although another study showed approximately no change in protein carbonylation across the age groups. Nitric oxide synthase (NOS) induces nitric oxide synthase (iNOS) inducible to produce NO. Increasing nitric oxide (NO) and nitric oxide synthase are promoted the repairment of damaged pathways, and accelerating endothelial nitric oxide synthase [90]. In this walking exercise training interventions regimens, inhibition of the extracellular-signal-regulated inducible nitric oxide synthase and downrequirement endothelial nitric oxide synthase (eNOS) resulting in disturbed RAS system. The ACE2-Ang III -AT1R/AT2R axis is a well-established component of RAS through Angiotensin (Ang $\Pi$ )/Angiotensin $I$ type 1 
receptor (AT1R) or Angiotensin (Ang $\Pi$ )/Angiotensin $I$ type 1 receptor (AT2R) $[91,92]$. Walking exercise training interventions improved cognitive remediation renin-angiotensin system (ARS) in the community older groups. After adaptive walking exercise training intervention with ketogenic diet for 2-rounds of walking exercise, the maximal exercise capacity test was measured (Table 2). Walking exercise training intervention after ketogenic diet activated SIRT-1/SIRT-3 signaling pathways [91,92-94] and vascular endothelial growth factor (VEGF) $[95,96]$, because walking exercise training interventions increased NAD/NADH ratio in the community older groups. SIRT-1/ SIRT-3 signaling pathways belong to renin-angiotensin system (ARS) have also been thoroughly explored $[97,98]$.

\section{Table 2: Histopathological and cellular markers found in the community older groups.}

Target

Modification

References

Angiotensin (Ang

II)/Ang type 1

receptor (AT1)

increased

$[90,91]$

Receptor for

advanced

glycation end

increased

[91-94]

product (RAGE)

B-amyloid

increased

[92-94]

RAS system

disrupted

[95]

SIRT-1/SIRT-3 pathway is a signaling pathway that preserves health under conditions demonstrated that the activation of AMPK through walking exercise training increases Sirt activation and mTOR inhibition [99]. Although walking exercise training is an effective way to improve SIRT-1, SIRT-3, VEGF, AMPK, and mTOR. Walking exercise training to regulate vascular endothelial growth 
factor (VEGF) and nitric oxide synthase (NOS) synthesis can rise various interventional. SIRT-1, SIRT-3, VEGF, AMPK, and mTOR are seen increases before and after our exercise intervention. NO and VEG has been demonstrated measurable decreases in the community older groups (Table 3 ). VEGF plays an important role in the benefits of walking exercise training performance and brain blood flow in the community older groups. The synthesis of VEGF can be induced by NO [92]. In addition, combined ketogenic diet and walking exercise training intervention were seen to increase intracellular AMPK pathway, the AMPK pathway was the main pathway through PI3K/Akt/mTOR pathway in the community older groups. Therefore, a walking exercise training was planned for up-regulation PI3K/Akt/mTOR and AMPK pathways and antiinflammatory $[100,102]$.

\section{Table 3: Molecular and cellular pathways altered in the community older groups.}

\begin{tabular}{lll}
\hline Target & Modification & References \\
\hline ACE2 & increased & {$[90,91]$} \\
SIRT-1 & decreased & {$[91-94]$} \\
SIRT-3 & decreased & {$[92-94]$} \\
VEGF & decreased & {$[95,96]$} \\
AMPK & decreased & {$[97,98]$} \\
mTOR & decreased & {$[99]$} \\
mtDNA & decreased & {$[100,101]$} \\
NF-KB & increased & {$[102]$} \\
\hline
\end{tabular}

Walking exercise training interventions generally leads to bred with mitochondrial DNA (mtDNA) affecting genes involved in every aspect of the mtDNA repair $[100,101]$. These findings combined are particularly interesting when considering mtDNA deletions and inflammation factor, NF-KB, in the community older groups.

\section{Conclusions}


Patients in the community older groups remain cooperative with the nutritional and walking exercise interventions will be reduced aging disorder diseases. In the older communication population, a walking exercise program improved healthy [102]. Some older communication patients reported mild no need intervention. Walking exercise interventions of shorter duration, no changes were observed for pre-and acclimation [103]. Most importantly, involving the use of accredited walking exercise physiologists implementing walking exercise programs for the community older groups. It should further be noted that walking exercise training programs and ketogenic diet interventions to the effective treatments for aging in the community older groups [104]. Exercise recommendations for the community older groups, the participants will conduct walking exercise training. The walking exercise was easy, not difficult. One other example exercise in the community older groups, ketogenic diet and walking exercise training intervention program for the patient was combined. This was associated with some improvement in molecular and cellular markers of the combined ketogenic diet and walking exercise training interventions in the community older groups performance [105]. This pragmatic trial in primary healthcare aimed to assess the effect of a health promotion program with or without exercise intervention on physical activity in community older groups. It is possible that exercise therapy has been reported to improve the walking distance sitting test, 6-min walking distance, and slow walking speed during walking periods. The content of the guidance used in the intervention has been effective in motivating subjects to exercise walking in the community older groups [106]. Exercise tolerance reduced already oxygen consumption during all those considered, walking training, resistance exercise and combined exercise is limited in the community older groups. It contrasts with its limited effect on exercise interventions, changes in vital signs during exercise, changes in energy metabolism, walking distance [107]. After each exercise regimen phase, we find ineligible interventions, especially during challenging walking conditions in the community older groups, such as the average walking speed for $15 \mathrm{~min}$ (Figure 2). For several decades, the role of the ketogenic diet in aging-related diseases has been known for thousands of years, and understanding the aging processes through the identification of gene variants to extend the lifespan of multicellular model organisms (Table 3). However, oxidative stress and ROS may induce the levels of protein carbonylation, oxidation, and lipid peroxidation products while causing the dysfunction of nucleic acids in the community older groups. 


\section{Acknowledgement:}

Not applicable.

\section{Funding:}

Not applicable.

\section{Conflicts of interest:}

The authors declare no conflicts of interest exist in the publication of this paper.

\section{Institutional Review Board Statement:}

Not applicable.

\section{Informed Consent Statement:}

Not applicable.

\section{Data Availability Statement:}

Data is available from the corresponding author upon reasonable request.

\section{References}

1. Jia, P.; Huang, B.; You, Y.; Su, H.; Gao, L. Ketogenic diet aggravates kidney dysfunction by exacerbating metabolic disorders and inhibiting autophagy in spontaneously hypertensive rats. Biochem Biophys Res Commun 2021, 573, 13-18.

2. Crosby, L.; Davis, B.; Joshi, S.; Jardine, M.; Paul, J.; Neola, M.; Barnard, N.D. Ketogenic Diets and Chronic Disease: Weighing the Benefits Against the Risks. Front Nutr 2021, 8, 702802.

3. Oosman, S.; Nisbet, C.; Smith, L.; Abonyi, S. Health promotion interventions supporting Indigenous healthy ageing: a scoping review. Int J Circumpolar Health 2021, 80, 1950391.

4. Andrianova, N.V.; Buyan, M.I.; Bolikhova, A.K.; Zorov, D.B.; Plotnikov, E.Y. Dietary Restriction for Kidney Protection: Decline in Nephroprotective Mechanisms During Aging. Front Physiol 2021, 12, 699490.

5. Sams, E.C. Oligodendrocytes in the aging brain. Neuronal Signal 2021, 5, NS20210008.

6. Turner, D.A. Contrasting Metabolic Insufficiency in Aging and Dementia. Aging Dis 2021, 12, 1081-1096. 
7. Kirmani, B.F.; Shapiro, L.A.; Shetty, A.K. Neurological and Neurodegenerative Disorders: Novel Concepts and Treatment. Aging Dis 2021, 12, 950-953.

8. Sefiani, A.; Geoffroy, C.G. The Potential Role of Inflammation in Modulating Endogenous Hippocampal Neurogenesis After Spinal Cord Injury. Front Neurosci 2021, 15, 682259.

9. Stephan, J.S.; Sleiman, S.F. Exercise Factors Released by the Liver, Muscle, and Bones Have Promising Therapeutic Potential for Stroke.

Front Neurol 2021, 12, 600365.

10. Alqurashi, R.S.; Yee, A.S.; Malone, T.; Alrubiaan, S.; Tam, M.W.; Wang, K.; Nandedwalla, R.R.; Field, W.; Alkhelb, D.; Given, K.S.; Siddiqui, R.; Baleja, J.D.; Paulson, K.E.; Yee, A.S. A Warburg-like metabolic program coordinates Wnt, AMPK, and mTOR signaling pathways in epileptogenesis. PLoS One 2021, 16, e0252282.

11. Al-Sofyani, K.A. An Insight into the Current Understanding of Status Epilepticus: From Concept to Management. Neurol Res Int 2021 2021, 9976754.

12. Wu, S.; Liu, X.; Jiang, R.; Yan, X.; Ling, Z. Roles and Mechanisms of Gut Microbiota in Patients With Alzheimer's Disease. Front Aging Neurosci 2021, 13, 650047.

13. Turner-McGrievy, G.M.; Jenkins, D.J.; Barnard, N.D.; Cohen, J.; Gloede, L.; Green, A.A. Decreases in dietary glycemic index are related to weight loss among individuals following therapeutic diets for type 2 diabetes. J Nutr 2011 141, 1469-1474.

14. Norwitz, N.G.; Winwood, R.; Stubbs, B.J.; D'Agostino, D.P.; Barnes, P.J. Case Report: Ketogenic Diet Is Associated With Improvements in Chronic Obstructive Pulmonary Disease. Front Med (Lausanne) 2021; 8:699427.

15. Sourbron, J.; Thevissen, K.; Lagae, L. The Ketogenic Diet Revisited: Beyond Ketones. Front Neurol 2021,12, 720073.

16. Tan, J.; Ni, D.; Wali, J.A.; Cox, D.A.; Pinget, G.V.; Taitz, J.; Daïen, Cl.; Senior, A.; Read, M.N.; Simpson, S.J.; King, N.J.C.; Macia, L. Dietary carbohydrate, particularly glucose, drives B cell lymphopoiesis and function. iScience 2021, 24, 102835.

17. Seira, O.; Kolehmainen, K.; Liu, J.; Streijger, F.; Haegert, A.; Lebihan, S.; Boushel, R.; Tetzlaff, W. Ketogenesis controls mitochondrial gene expression and rescues mitochondrial bioenergetics after cervical spinal cord injury in rats. Sci Rep 2021, 11, 16359.

18. Seo, Y.G.; Oh, S.; Park, W.H.; Jang, M.; Kim, H.Y.; Chang, S.A.; Park, 
I.K.; Sung, J. Optimal aerobic exercise intensity and its influence on the effectiveness of exercise therapy in patients with pulmonary arterial hypertension: a systematic review. J Thorac Dis 2021, 13, 4530-4540.

19. O'Mara, S. Biopsychosocial Functions of Human Walking and Adherence to Behaviourally Demanding Belief Systems: A Narrative Review. Front Psychol, 2021, 12, 654122.

20. Koziel, N.; Vigod, S.; Price, J.; Leung, J.; Hensel, J. Walking Psychotherapy As a Health Promotion Strategy to Improve Mental and Physical Health for Patients and Therapists: Clinical Open-Label Feasibility Trial. Can J Psychiatry, 2021, 7067437211039194.

21. Aoi, S.; Amano, T.; Fujiki, S.; Senda, K.; Tsuchiya, K. Fast and Slow Adaptations of Interlimb Coordination via Reflex and Learning During SplitBelt Treadmill Walking of a Quadruped Robot. Front Robot Al, 2021, 8, 697612.

22. Wu, J.; Zhao, C.; Li, C.; Wang, T.; Wang, L.; Zhang, Y. Non-linear Relationships Between the Built Environment and Walking Frequency Among Older Adults in Zhongshan, China. Front Public Health 2021, 9, 686144.

23. Buurke, T.J.W.; den Otter, R. The relationship between the anteroposterior and mediolateral margins of stability in able-bodied human walking. Gait Posture 2021, 90, 80-85.

24. Fallahtafti, F.; Gonabadi, A.M.; Samson, K.; Yentes, J.M. Margin of Stability May Be Larger and Less Variable during Treadmill Walking Versus Overground. Biomechanics (Basel) 2021, 1, 118-130.

25. Dhungana, R.K.; Sapkota, R.R.; Niroula, D.; Giri, R. Walking metals: catalytic difunctionalization of alkenes at nonclassical sites. Chem Sci 2020, 11, 9757-9774.

26. Augustin, J.; Boivin, G.; Brodeur, J.; Bourgeois, G. Effect of temperature on the walking behaviour of an egg parasitoid: disentangling kinetic response from integrated response. Ecol Entomol 2020, 45, 741-750.

27. Altimir, C.; Jimenez, J.P. Walking the middle ground between hermeneutics and science: A research proposal on psychoanalytic process. Int J Psychoanal 2020, 101, 496-522.

28. Miller, B.C.; Tirko, A.W.; Shipe, J.M.; Sumeriski, O.R.; Moran, K. The Systemic Effects of Blood Flow Restriction Training: A Systematic Review. Int J Sports Phys Ther 2021, 16, 978-990.

29. Swanson, R.; Robinson, K.M. Geriatric Rehabilitation: Gait in the Elderly, Fall Prevention and Parkinson Disease. Med Clin North Am 2019, 104, 
327-343.

30. Campisi, J.; Kapahi, P.; Lithgow, G.J.; Melov, S.; Newman, J.C.; Verdin, E. From discoveries in ageing research to therapeutics for healthy ageing. Nature 2019, 571, 183-192.

31. Grammatikopoulou, M.G.; Goulis, D.G.; Gkiouras, K.; Theodoridis, X.; Gkouskou, K.K.; Evangeliou, A.; Dardiotis, E.; Bogdanos, D.P. To Keto or Not to Keto? A Systematic Review of Randomized Controlled Trials Assessing the Effects of Ketogenic Therapy on Alzheimer Disease. Adv Nutr 2020, 11, 1583-1602.

32. Cavaleri, F.; Bashar, E. Potential Synergies of $\beta$-Hydroxybutyrate and Butyrate on the Modulation of Metabolism, Inflammation, Cognition, and General Health. J Nutr Metab 2018, 2018, 7195760.

33. Oliveira, R.C.; Pralle, R.S.; de Resende, L.C.; Nova, C.H.P.C.; Caprarulo, V.; Jendza, J.A.; Troescher, A.; White, H.M. Prepartum supplementation of conjugated linoleic acids (CLA) increased milk energy output and decreased serum fatty acids and $\beta$-hydroxybutyrate in early lactation dairy cows. PLoS One 2018, 13, e0197733.

34. Caminhotto, R.O.; Komino, A.C.M.; de Fatima Silva, F.; Andreotti, S.; Sertié R.A.L.; Boltes Reis, G.; Lima, F.B. Oral $\beta$ hydroxybutyrate increases ketonemia, decreases visceral adipocyte volume and improves serum lipid profile in Wistar rats. Nutr Metab (Lond) 2017, 14, 31.

35. Chawla, R.; Madhu, S.V.; Makkar, B.M.; Ghosh, S.; Saboo, B.; Kalra, S. RSSDI-ESI Consensus Group RSSDI-ESI Clinical Practice Recommendations for the Management of Type 2 Diabetes Mellitus 2020. Indian J Endocrinol Metab 2020, 24, 1-122.

36. Smith, P.J. Pathways of Prevention: A Scoping Review of Dietary and Exercise Interventions for Neurocognition. Brain Plast 2019, 5, 3-38.

37. Bray, G.A.; Heisel, W.E.; Afshin, A.; Jensen, M.D.; Dietz, W.H.; Long, M.; Kushner, R.F.; Daniels, S.R.; Wadden, T.A.; Tsai, A.G.; Hu, F.B.; Jakicic, J.M.; Ryan, D.H.; Wolfe, B.M.; Inge, T.H. The Science of Obesity Management: An Endocrine Society Scientific Statement. Endocr Rev 2018, 39, 79-132.

38. Naude, C.E.; Visser, M.E.; Nguyen, K.A.; Durao, S.; Schoonees, A. Effects of total fat intake on bodyweight in children. Cochrane Database Syst Rev 2018, 2, CD012960.

39. Al-Khudairy, L.; Loveman, E.; Colquitt, J.L.; Mead, E.; Johnson, R.E.; Fraser, H.; Olajide, J.; Murphy, M.; Velho, R.M.; O'Malley, 
C.; Azevedo, L.B.; Ells, L.J.; Metzendorf, M.I.; Rees, K. Diet, physical activity and behavioural interventions for the treatment of overweight or obese adolescents aged 12 to 17 years. Cochrane Database Syst Rev 2017, 6, CD012691.

40. Nehls, M. Unified theory of Alzheimer's disease (UTAD): implications for prevention and curative therapy. J Mol Psychiatry 2016, 4, 3.

41. Stacey, F.G.; James, E.L.; Chapman, K.; Courneya, K.S.; Lubans, D.R. A systematic review and meta-analysis of social cognitive theory-based physical activity and/or nutrition behavior change interventions for cancer survivors. J Cancer Surviv 2014, 9, 305-338.

42. Minich, D.M.; Bland, J.S. Personalized lifestyle medicine: relevance for nutrition and lifestyle recommendations. ScientificWorldJournal 2013, 2013, 129841.

43. Morgan, A.J.; Jorm, A.F. Self-help interventions for depressive disorders and depressive symptoms: a systematic review. Ann Gen Psychiatry 2008, $7,13$.

44. George, M.; Topaz, M. A systematic review of complementary and alternative medicine for asthma self-management. Nurs Clin North Am 2013, 48, 53-149.

45. Norgren, J.; Daniilidou, M.; Kåreholt, I.; Sindi, S.; Akenine, U.; Nordin, K.; Rosenborg, S.; Ngandu, T.; Kivipelto, M.; Sandebring-Matton, A. Serum proBDNF Is Associated With Changes in the Ketone Body $\beta$ Hydroxybutyrate and Shows Superior Repeatability Over Mature BDNF: Secondary Outcomes From a Cross-Over Trial in Healthy Older Adults. Front Aging Neurosci 2021, 13, 716594.

46. Simpson, D.J.; Olova, N.N.; Chandra, T. Cellular reprogramming and epigenetic rejuvenation. Clin Epigenetics 2021, 13, 170.

47. Karlstaedt, A.; Barrett, M.; Hu, R.; Gammons, S.T.; Ky, B. Cardio-Oncology: Understanding the Intersections Between Cardiac Metabolism and Cancer Biology. JACC Basic Transl Sci 2021, 6, 705-718.

48. Westman, E.C. Type 2 Diabetes Mellitus: A Pathophysiologic Perspective. Front Nutr 2021, 8, 707371.

49. Di Raimondo, D.; Buscemi, S.; Musiari, G.; Rizzo, G.; Pirera, E.; Corleo, D.; Pinto, A.; Tuttolomondo, A. Ketogenic Diet, Physical Activity, and Hypertension-A Narrative Review. Nutrients 2021, 13.

50. Yao, A.; Li, Z.; Lyu, J.; Yu, L.; Wei, S.; Xue, L.; Wang, H.; Chen, G.Q. On the nutritional and therapeutic effects of ketone body D- $\beta$-hydroxybutyrate. Appl Microbiol Biotechnol 2021, 105, 6229-6243. 
51. Mann, G.; Mora, S.; Madu, G.; Adegoke, O.A.J. Branched-chain Amino Acids: Catabolism in Skeletal Muscle and Implications for Muscle and Whole-body Metabolism. Front Physiol 2021, 12, 702826.

52. Koronowski, K.B.; Greco, C.M.; Huang, H.; Kim, J.K.; Fribourgh, J.L.; Crosby, P.; Mathur, L.; Ren, X.; Partch, C.L.; Jang, C.; Qiao, F.; Zhao, Y.; Sassone-Corsi, P. Ketogenesis impact on liver metabolism revealed by proteomics of lysine $\beta$-hydroxybutyrylation. Cell Rep 2021, 36, 109487.

53. Zhao, Y.; Pang, D.; Lu, Y. The Role of Nurse in the Multidisciplinary Management of Cancer Cachexia. Asia Pac J Oncol Nurs 2021, 8, 487497.

54. Hopkinson, J.B. The Psychosocial Components of Multimodal Interventions Offered to People with Cancer Cachexia: A Scoping Review. Asia Pac J Oncol Nurs 2021, 8, 450-461.

55. Yang, S.S.; Seo, T.B.; Kim, Y.P. Effect of aqua walking exercise on knee joint angles, muscular strength, and visual analogue scale for patients with limited range of motion of the knee. J Exerc Rehabil 2021, 17, 265-269.

56. Kanegusuku, H.; Ritti-Dias, R.M.; Barbosa, P.Y.I.; das Neves Guelfi, E.T.; Okamoto, E.; Miranda, C.S.; de Paula Oliveira, T.; Piemonte, M.E.P. Influence of motor impairment on exercise capacity and quality of life in patients with Parkinson disease. J Exerc Rehabil 2021, 17, 241-246.

57. Becker, K.; Uebing, A.; Hansen, J.H. Pulmonary vascular disease in Fontan circulation-is there a rationale for pulmonary vasodilator therapies? Cardiovasc Diagn Ther 2021, 11, 1111-1121.

58. Xiang, G.; Zhu, X.; Ma, L.; Huang, H.; Wu, X.; Zhang, W.; Li, S. Clinical guidelines on the application of Internet of Things (IOT) medical technology in the rehabilitation of chronic obstructive pulmonary disease.

$\mathrm{J}$ Thorac Dis 2021, 13, 4629-4637.

59. Özer, F.F.; Akin, S.; Gültekin, M.; Zararsiz, G.E.; Soylu, A.E. Frailty in Patients with Parkinson's Disease: Associations with Disability and Timed Up and Go. Noro Psikiyatr Ars 2019, 58, 206-212.

60. OTSUKA, S.; MORISAWA, T.; HOJO, Y.; ISHIDA, A.; TAMAKI, A. Effect of Home-based Exercise Therapy for Peripheral Arterial Disease Patients Underwent Endovascular Treatment: A Clinical Controlled Design. Phys Ther Res 2021, 24, 120-127.

61. Grigoletto, A.; Mauro, M.; Maietta Latessa, P.; lannuzzi, V.; Gori, D.; Campa, F.; Greco, G.; Toselli, S. Impact of Different Types of Physical Activity in Green Urban Space on Adult Health and Behaviors: A Systematic Review. Eur J Investig Health Psychol Educ 2021, 11, 263-275. 
62. Marais, G.; Lantheaume, S.; Fiault, R.; Shankland, R. Mindfulness-Based Programs Improve Psychological Flexibility, Mental Health, Well-Being, and Time Management in Academics. Eur J Investig Health Psychol Educ 2020, 10, 1035-1050.

63. Verghese, J.; Mahoney, J.R.; Ayers, E.; Ambrose, A.; Wang, C. Holtzer, R. Computerised cognitive remediation to enhance mobility in older adults: a single-blind, single-centre, randomised trial. Lancet Healthy Longev 2021, 2, e571-e579.

64. Schladen, M.M.; Cleary, K.; Koumpouros, Y.; Monfaredi, R.; Salvador, T.; Talari, H.F.; Slagle, J.; Coley, C.; Kovelman, S.; Belschner, J.; Evans, S.H. Toward Evaluation of the Subjective Experience of a General Class of User-Controlled, Robot-Mediated Rehabilitation Technologies for Children with Neuromotor Disability. Informatics (MDPI) 2020, 7, 45-50.

65. Koh, F.H.; Chua, J.M.; Tan, J.L.; Foo, F.J.; Tan, W.J.; Sivarajah, S.S.; Ho, L.M.L.; Teh, B.T.; Chew, M.H. Paradigm shift in gastrointestinal surgery combating sarcopenia with prehabilitation: Multimodal review of clinical and scientific data. World J Gastrointest Surg 2021, 13, 734-755.

66. Davoodi, M.; Zilaei, B.S.; Dehghan, G.S. Antioxidant Effects of Aerobic Training and Crocin Consumption on Doxorubicin-Induced Testicular Toxicity in Rats. J Family Reprod Health 2021, 15, 28-37.

67. Zhou, D.D.; Luo, M.; Huang, S.Y.; Saimaiti, A.; Shang, A.; Gan, R.Y.; Li, H.B. Effects and Mechanisms of Resveratrol on Aging and Age-Related Diseases. Oxid Med Cell Longev 2021, 2021, 9932218.

68. Cüzdan, N.; Türk, İ.; Çiftçi, V.; Arslan, D.; Doğan, M.C.; Ünal, İ. The effect of a home-based orofacial exercise program on oral aperture of patients with systemic sclerosis: A single-blind prospective randomized controlled trial. Arch Rheumatol 2021, 36, 176-184.

69. Beak, M.; Choi, W.J.; Lee, W.; Ham, S. Associations of Abnormal Sleep Duration with Occupational and Leisure-time Physical Activity in the Working Population: A Nation-wide Population-based Study. Saf Health Work 2021, 12, 311-316.

70. Ruksakulpiwat, S.; Zhou, W. Self-management interventions for adults with stroke: A scoping review. Chronic Dis Transl Med 2021, 7, 139-148.

71. Rubfiaro, A.S.; Tsegay, P.S/; Lai, Y.; Cabello, E.; Shaver, M.; Hutcheson, J.; Liu, Y.; He, J. Scanning Ion Conductance Microscopy Study Reveals the Disruption of the Integrity of the Human Cell Membrane Structure by Oxidative DNA Damage. ACS Appl Bio Mater 2021, 4, 1632-1639.
72. Álvarez-Satta,
M.; Berna-Erro,
A.; Carrasco-Garcia,
E.; Alberro, 
A.; Saenz-Antoñanzas, A.; Vergara, I.; Otaegui, D.; Matheu, A. Relevance of oxidative stress and inflammation in frailty based on human studies and mouse models. Aging (Albany NY) 2020, 12, 9982-9999.

73. Angulo, J.; El Assar, M.; Álvarez-Bustos, A.; Rodríguez-Mañas, L. Physical activity and exercise: Strategies to manage frailty. Redox Biol 2020, 35 , 101513.

74. Spanidis, Y.; Stagos, D.; Papanikolaou, C.; Karatza, K.; Theodosi, A.; Veskoukis, A.S.; Deli, C.K.; Poulios, A.; Koulocheri, S.D.; Jamurtas, A.Z.; Haroutounian, S.A.; Kouretas, D. Resistance-Trained Individuals Are Less Susceptible to Oxidative Damage after Eccentric Exercise. Oxid Med Cell Longev 2018, 2018, 6857190.

75. Wu, G. Important roles of dietary taurine, creatine, carnosine, anserine and 4-hydroxyproline in human nutrition and health. Amino Acids, 2020, 52, 329-360.

76. Williamson, E. Nutritional implications for ultra-endurance walking and running events. Extrem Physiol Med, 2016, 5, 13.

77. Sallam, N.; Laher, I. Exercise Modulates Oxidative Stress and Inflammation in Aging and Cardiovascular Diseases. Oxid Med Cell Longev 2015, 2016, 7239639.

78. Phillip, J.M.; Aifuwa, I.; Walston, J.; Wirtz, D. The Mechanobiology of Aging. Annu Rev Biomed Eng 2015, 17, 113-141.

79. Lee, M.C.; Hsu, Y.J.; Ho, C.S.; Chang, C.H.; Liu, C.W.; Huang, C.C.; Chiang, W.D. Evaluation of the Efficacy of Supplementation with Planox $®$ Lemon Verbena Extract in Improving Oxidative Stress and Muscle Damage: A Randomized Double-Blind Controlled Trial. Int J Med Sci 2021, 18, 2641-2652.

80. Lu, Y.; Niti, M.; Yap, K.B.; Tan, C.T.Y.; Nyunt, M.S.Z.; Feng, L.; Tan, B.Y.; Chan, G.; Khoo, S.A.; Chan, S.M.; Yap, P.; Larbi, A.; Ng, T.P. Effects of multi-domain lifestyle interventions on sarcopenia measures and blood biomarkers: secondary analysis of a randomized controlled trial of community-dwelling pre-frail and frail older adults. Aging (Albany NY) 2021, 13, 9330-9347.

81. Kruk, J.; Aboul-Enein, B.H.; Duchnik, E. Exercise-induced oxidative stress and melatonin supplementation: current evidence. J Physiol Sci 2021, 71, 27.

82. Schätzl, T.; Kaiser, L.; Deigner, H.P. Facioscapulohumeral muscular dystrophy: genetics, gene activation and downstream signalling with regard to recent therapeutic approaches: an update. Orphanet J Rare Dis 
2021, 16, 129.

83. Guerrero, C.; Collado-Boira, E.; Martinez-Navarro, I.; Hernando, B.; Hernando, C.; Balino, P.; Muriach, M. Impact of Plasma Oxidative Stress Markers on Post-race Recovery in Ultramarathon Runners: A Sex and Age Perspective Overview. Antioxidants (Basel) 2021, 10.

84. Dara, A.; Arvanitaki, A.; Theodorakopoulou, M.; Athanasiou, C.; Pagkopoulou, E.; Boutou, A. Non-Invasive Assessment of Endothelial Dysfunction in Pulmonary Arterial Hypertension. Mediterr J Rheumatol 2021, 32, 6-14.

85. Hurşitoğlu, O.; Orhan, F.Ö.; Kurutaş, E.B.; Doğaner, A.; Durmuş, H.T.; Kopar, H. Diagnostic Performance of Increased Malondialdehyde Level and Oxidative Stress in Patients with Schizophrenia. Noro Psikiyatr Ars 2021, 58, 184-188.

86. Bian, J.; Li, Z. Angiotensin-converting enzyme 2 (ACE2): SARS-CoV2 receptor and RAS modulator. Acta Pharm Sin B 2020, 11, 1-12.

87. Wang, D.; Cao, H.; Wang, X.; Wang, J.; Wang, M.; Zhang, J.; Wang, L. SIRT1 is Required for Exercise-Induced Beneficial Effects on Myocardial Ischemia/Reperfusion Injury. J Inflamm Res 2021, 14, 1283-1296.

88. Askin, L.; Tibilli, H.; Tanriverdi, O.; Turkmen, S. The relationship between coronary artery disease and SIRT1 protein. North Clin Istanb 2020, 7, 631635.

89. Du, X.; Chen, W.; Zhan, N.; Bian, X.; Yu, W. The effects of low-intensity resistance training with or without blood flow restriction on serum BDNF, VEGF and perception in patients with post-stroke depression. Neuro Endocrinol Lett 2021, 42, 229-235.

90. Fu, P.; Zhu, R.; Jia, J.; Hu, Y.; Wu, C.; Cieszczyk, P.; Holmberg, H.C.; Gong, L. Aerobic exercise promotes the functions of brown adipose tissue in obese mice via a mechanism involving COX2 in the VEGF signaling pathway. Nutr Metab (Lond), 2021, 18, 56.

91. Hu, H.; Xia, N.; Lin, J.; Li, D.; Zhang, C.; Ge, M.; Tian, H.; Mei, X. Zinc Regulates Glucose Metabolism of the Spinal Cord and Neurons and Promotes Functional Recovery after Spinal Cord Injury through the AMPK Signaling Pathway. Oxid Med Cell Longev 2021, 2021, 4331625.

92. Maharajan, N.; Ganesan, C.D.; Moon, C.; Jang, C.H.; Oh, W.K.; Cho, G.W. Licochalcone D Ameliorates Oxidative Stress-Induced Senescence via AMPK Activation. Int J Mol Sci 2021, 22.

93. Ritz, A.; Froeba-Pohl, A.; Kolorz, J.; Vigodski, V.; Hubertus, J.; LeyZaporozhan, J.; von Schweinitz, D.; Häberle, B.; Schmid, I.; Kappler, 
R.; Lurz, E.; Berger, M. Total Psoas Muscle Area as a Marker for Sarcopenia Is Related to Outcome in Children With Neuroblastoma. Front Surg 2021, 8, 718184.

94. Sellami, M.; Bragazzi, N.; Prince, M.S.; Denham, J.; Elrayess, M. Regular, Intense Exercise Training as a Healthy Aging Lifestyle Strategy: Preventing DNA Damage, Telomere Shortening and Adverse DNA Methylation Changes Over a Lifetime. Front Genet 2021, 12, 652497.

95. Li, Z.; Huang, Z.; Zhang, H.; Lu ,J.; Wei, Y.; Yang, Y.; Bai, L. IRE1-mTORPERK Axis Coordinates Autophagy and ER Stress-Apoptosis Induced by P2X7-Mediated $\mathrm{Ca}^{2+}$ Influx in Osteoarthritis. Front Cell Dev Biol 2021, 9, 695041.

96. Wen, C.; Ying, Y.; Zhao, H.; Jiang, Q.; Gan, X.; Wei, Y.; Wei, J.; Huang, X. Resistance exercise affects catheter-related thrombosis in rats through miR-92a-3p, oxidative stress and the MAPK/NF-kB pathway. BMC Cardiovasc Disord 2021, 21, 440.

97. Jevtovic, F. Combination of Metformin and Exercise in Management of Metabolic Abnormalities Observed in Type 2 Diabetes Mellitus. Diabetes Metab Syndr Obes 2021,14,4043-4057.

98. Feike, Y.; Zhijie, L.; Wei, C. Advances in research on pharmacotherapy of sarcopenia. Aging Med (Milton) 2021, 4, 221-233.

99. Ou, Y.; Zhang, W.; Chen, S.; Deng, H. Baicalin improves podocyte injury in rats with diabetic nephropathy by inhibiting PI3K/Akt/mTOR signaling pathway. Open Med (Wars) 2021, 16, 1286-1298.

100.Melicher, D.; Illés, A.; Littvay, L.; Tárnoki, Á.D.; Tárnoki, D.L.; Bikov, A.; Kunos, L.; Csabán, D.; Buzás, E.I.; Molnár, M.J.; Falus, A. Positive association and future perspectives of mitochondrial DNA copy number and telomere length - a pilot twin study. Arch Med Sci 2019, 17, 11911199.

101.Baek, K.W.; Jung, Y.K.; Park, J.S.; Kim, J.S.; Hah, Y.S.; Kim, S.J.; Yoo, J.I. Two Types of Mouse Models for Sarcopenia Research: Senescence Acceleration and Genetic Modification Models. J Bone Metab 2021, 28, 179-191.

102.Nikniaz, L.; Ghojazadeh, M.; Nateghian, H.; Nikniaz, Z.; Farhangi, M.A.; Pourmanaf, $H$. The interaction effect of aerobic exercise and vitamin $\mathrm{D}$ supplementation on inflammatory factors, anti-inflammatory proteins, and lung function in male smokers: a randomized controlled trial. BMC Sports Sci Med Rehabil 2021, 13, 102.

103.Jia, N.; Zhou, Y.; Dong, X.; Ding, M. The antitumor mechanisms of 
aerobic exercise: A review of recent preclinical studies. Cancer Med 2021, 10, 6365-6373.

104.Oo, Z.; Bhavsar, D.; Aung, T.; Ayala-Rodriguez, C.; Kyaw, H.

Exercise Stress Test-Induced Atrioventricular Dissociation With Syncope. Ochsner J 2021, 21, 319-324.

105.JAKOBSSON, J.; THEOS, A.; MALM, C. Effects of Different Types of Lower Body Resistance Exercise on Upper-body Strength in Men and Women, with Special Reference to Anabolic Hormones. Int J Exerc Sci 2021, 14, 1052-1069.

106.Romero-Franco, N.; Molina-Mula, J.; Bosch-Donate, E.; Casado, A. Therapeutic exercise to improve pelvic floor muscle function in a female sporting population: a systematic review and meta-analysis. Physiotherapy 2021, 113, 44-52.

107.Rahmati, M.; Malakoutinia, F. Aerobic, resistance and combined exercise training for patients with amyotrophic lateral sclerosis: a systematic review and meta-analysis. Physiotherapy 2021, 113, 12-28. 